\title{
El papel del género en la demanda de atención por problemas asociados al consumo de alcohol en México
}

\author{
Mariana Belló, ${ }^{1}$ Esteban Puentes-Rosas ${ }^{1}$ \\ y María Elena Medina-Mora Icaza²
}

Forma de citar

Belló M, Puentes-Rosas E, Medina-Mora Icaza ME. El papel del género en la demanda de atención por problemas asociados al consumo de alcohol en México. Rev Panam Salud Publica. 2008;23(4):231-6.

RESUMEN Objetivos. Actualizar la información sobre el papel del género en la búsqueda de ayuda por parte de consumidores de alcohol, así como evaluar las asociaciones de la demanda con otras variables de interés.

Métodos. La información proviene de la Encuesta Nacional de Adicciones 2002, que incluyó 11252 hogares, en los cuales se entrevistó a un adulto entre 12 y 65 años de edad. Los resultados de este estudio se basan en las respuestas de las 5406 personas identificadas como consumidoras de alcohol. La demanda de atención se definió como la búsqueda de ayuda por problemas de consumo en el último año. Los consumidores de alcohol quedaron definidos como aquellas personas que consumieron alcohol en el último año. Se calculó la prevalencia nacional de búsqueda de ayuda según género, patrones de consumo, síndrome de dependencia y otros problemas relacionados con el consumo de alcohol. Se realizaron regresiones logísticas utilizando la demanda de atención como variable dependiente.

Resultados. De los bebedores, 1,4\% solicitaron ayuda debido a sus problemas de consumo de alcohol. El porcentaje correspondiente en hombres fue de 2,1\%, mientras que en las mujeres fue de $0,2 \%(\mathrm{P}<0,001)$. Por cada mujer dependiente que solicita ayuda, hay 49 hombres que lo hacen. En el análisis multivariado, el género aparece asociado significativamente -usando a las mujeres como referencia-, aún controlando por dependencia y por haber perdido el trabajo debido al consumo de alcohol.

Conclusiones. Los hombres tienen mayor probabilidad de solicitar servicios de atención en comparación con las mujeres, quienes enfrentan obstáculos de tipo cultural, familiar y de los servicios. Otro importante predictor es el hecho de haber perdido el trabajo por la bebida. Es necesario enfocar más eficazmente la planeación de servicios a través de programas diseñados para atender las necesidades específicas de las mujeres.

Palabras clave Servicios de salud mental, trastornos relacionados con alcohol, género, México.

El abuso en el consumo de alcohol en México representa uno de los pro-

\footnotetext{
Dirección General de Evaluación del Desempeño, Secretaría de Salud, México. La correspondencia deberá enviarse a Mtra. Mariana Belló, Paseo del Río 300, Colonia Paseos de Taxqueña, Delegación Coyoacán, CP 04250, México, D.F., México. T.: 00.52. 55.56.70.71.62 Correo electrónico: bellomar@ hotmail.com

2 Instituto Nacional de Psiquiatría "Ramón de la Fuente Muñiz", México.
}

blemas de salud pública más importantes debido a sus elevados costos en los planos individual, familiar y social, y por su impacto en el sistema de salud. En la literatura nacional e internacional (1-5) se ha notificado consistentemente el hecho de que, a pesar de su importancia, la demanda de atención por consumo de alcohol es muy reducida.
Si bien la literatura sobre el tema muestra que la variable más frecuentemente relacionada con la búsqueda de atención es la percepción de consecuencias adversas relacionadas con el consumo de alcohol (6-13), el género aparece de manera destacada y consistente a lo largo de varios estudios, observándose que la proporción de mujeres que solicitan tratamiento por los problemas 
derivados del consumo siempre es mucho menor a la de los hombres $(2,5$, $6,14-17)$. Lo anterior se aprecia independientemente de la frecuencia con que se presentan problemas asociados con el consumo de alcohol (18).

En este mismo sentido, Weisner y cols. (2) analizaron las tendencias en cuanto al uso de servicios por problemas relacionados con el consumo de alcohol en población general, en los años 1979, 1984 y 1990, y notificaron que los varones tuvieron contacto con servicios de tratamiento en mayor proporción que las mujeres. Entre los aspectos más destacados de este estudio se encuentra el marcado efecto que el género tuvo con la probabilidad de buscar servicios de atención, incluso después de controlar por variables relacionadas con problemas por el consumo de alcohol.

Kaskutas y cols. (17) encontraron resultados similares al investigar el comportamiento de búsqueda de ayuda en una muestra de población general. Mediante un panel de individuos entrevistados en 1984, y posteriormente en 1992, hallaron que la probabilidad de que los hombres buscaran ayuda era dos veces mayor que en el caso de las mujeres.

En México se han llevado a cabo estudios para explorar la prevalencia de utilización de servicios, así como algunas características de la conducta de búsqueda de ayuda, componentes de redes sociales y preferencias institucionales para la utilización.

Con datos de la Encuesta Nacional de Adicciones y la Encuesta Nacional de Salud Mental, ambas del año 1988, Solís y Medina-Mora (15) exploraron los patrones de utilización de los servicios de salud mental que hacían las mujeres y encontraron que, mientras 3,2\% de los hombres solicitaron ayuda a un médico, un hospital o a otras instituciones por consumo de alcohol, el equivalente en las mujeres era de $0,8 \%$. Dado el incremento en el consumo de alcohol en las mujeres, aunado a los cambios en los roles de género, es probable que esta situación se haya modificado.

Este artículo tiene como objetivo actualizar la información sobre el rol del género en la búsqueda de ayuda, así como evaluar sus posibles asociaciones con otras variables identificadas como determinantes de la búsqueda de atención.

\section{MATERIAL Y MÉTODOS}

La fuente de información para la realización de este trabajo fue la Encuesta Nacional de Adicciones (ENA) 2002, cuyo trabajo de campo se realizó entre febrero y mayo de 2002. El diseño muestral de la encuesta fue probabilístico, estratificado, polietápico y por conglomerados, utilizando como unidad primaria de muestreo las Áreas Geoestadísticas Básicas (AGEB) de México (19). El número total de hogares encuestados fue de 11252 y en cada uno se seleccionó un informante de 12 a 65 años de edad, mediante un procedimiento aleatorio. Los resultados presentados en este trabajo se basan en la información proporcionada por 5406 personas, identificadas en este estudio como bebedores.

Los datos se recabaron mediante la aplicación de un cuestionario estandarizado, diseñado para ser administrado en una entrevista cara a cara. Los indicadores y preguntas que conforman este cuestionario han sido validados en estudios previos (20-22). Para el operativo de campo, el personal fue capacitado por un equipo especializado de instructores (19).

El cuestionario contiene indicadores propuestos por la Organización Mundial de la Salud para evaluar uso/ abuso y dependencia de sustancias, así como problemas relacionados. Incluye, además, preguntas que identifican al usuario de acuerdo con variables sociodemográficas y demanda de atención.

La variable de interés fue denominada demanda de atención y se definió como la búsqueda o solicitud de ayuda a alguna institución o persona, para consultar por problemas relacionados con el consumo de bebidas alcohólicas en los últimos 12 meses.

Bajo esta definición, se calcularon las prevalencias nacionales según género, patrones de consumo, síndrome de dependencia y otros problemas relaciona- dos con el consumo de alcohol. El patrón de consumo se definió como el comportamiento o modalidad de consumo de las personas y se construyó con datos de la frecuencia con que se consumen diferentes cantidades de alcohol por ocasión de consumo (22). Así, los consumidores pueden ubicarse en categorías que van desde bebedor poco frecuente de bajo nivel, hasta bebedor frecuente consuetudinario. La categorización de patrones de consumo derivados de este índice asume que una persona que bebe grandes cantidades de alcohol por ocasión de consumo, tendrá más problemas que una persona que limita la cantidad a una o dos copas, aunque ésta última beba con más frecuencia (23). Con base en esto, esta variable se organizó considerando desde los patrones de consumo de menor riesgo a los de mayor riesgo, de la siguiente forma: 1) bebedor poco frecuente de bajo nivel; 2) bebedor moderado de bajo nivel; 3 ) bebedor frecuente de bajo nivel; 4) bebedor poco frecuente de alto nivel; 5) bebedor moderado de alto nivel; 6) bebedor frecuente de alto nivel y 7) bebedor consuetudinario.

El síndrome de dependencia al alcohol se definió como el conjunto de manifestaciones fisiológicas, conductuales y cognitivas en el cual el consumo de bebidas alcohólicas adquiere la máxima prioridad para el individuo, mayor incluso que cualquier otro comportamiento que en el pasado tenía un gran valor para la persona (24). La presencia del síndrome se determinó mediante una serie de reactivos, evaluados como experiencias ocurridas en los últimos 12 meses. La presencia de, por lo menos, tres de los siguientes síntomas evaluados mediante estos reactivos, indica dependencia del alcohol: i) tolerancia; ii) abstinencia; iii) uso por más tiempo o en mayor cantidad; iv) deseo persistente; v) empleo de mucho tiempo para conseguir alcohol o para recuperarse de sus efectos; vi) reducción de actividades sociales para conseguir alcohol o recuperarse de sus efectos, y vii) uso continuo de alcohol a pesar de tener conciencia de problemas psicológicos o físicos.

Los problemas sociales relacionados se definieron como notificaciones posi- 
tivas de consecuencias negativas derivadas del consumo de alcohol en el bienestar social del individuo consumidor. A este respecto, se indagó acerca de: i) problemas con las autoridades mientras consumía bebidas alcohólicas aunque no estuviera conduciendo; ii) arresto mientras conducía después de tomar; iii) pérdida del empleo debido a la bebida y, finalmente, iv) discusión o pelea con el (la) esposo(a), compañero(a) cuando estuvo tomando.

En primera instancia se realizó un análisis bivariado para calcular la asociación entre estas variables independientes y la demanda de atención. Posteriormente, se llevaron a cabo regresiones logísticas utilizando esta última variable como dependiente, y las variables sociodemográficas, de consumo y de problemas relacionados, como independientes. Las variables incorporadas al modelo logístico fueron aquellas que conceptual o estadísticamente mostraron asociación con la probabilidad de buscar atención. En todos los casos se tomó en cuenta el procedimiento de muestreo complejo para ponderar las observaciones y para estimar la varianza compuesta de las estimaciones.

\section{RESULTADOS}

Del total de entrevistados, $46.3 \%$ refirieron haber consumido alcohol en el año previo a la encuesta; la mayoría fueron hombres $(59,3 \%)$. De aquí en adelante se hará referencia a ellos como "bebedores".

En lo que respecta al patrón de consumo, gran parte de los bebedores se ubican en categorías de consumo de bajo riesgo; no obstante, cabe destacar que $10 \%$ de los bebedores, consumen alcohol de manera consuetudinaria (cuadro 1). Las mujeres bebedoras se ubican con más frecuencia en las categorías de consumo de menor riesgo.

Poco menos de $10 \%$ de los entrevistados refirió tener tres o más síntomas para la definición del síndrome de dependencia al alcohol. De éstos, 13,5\% eran hombres y $1,9 \%$ mujeres, lo cual representa una diferencia estadísticamente significativa $(P<0,001)$.

CUADRO 1. Características de los bebedores según variables de consumo y problemas relacionados, México, 2002

\begin{tabular}{lccc}
\hline \multicolumn{1}{c}{ Variable } & $\begin{array}{c}(\%) \\
\text { Hombres }^{\mathrm{a}} \\
(n=3182)\end{array}$ & $\begin{array}{c}(\%) \\
\text { Mujeres }^{\mathrm{a}} \\
(n=2224)\end{array}$ & $\begin{array}{c}(\%) \\
\text { Total } \\
(n=5406)\end{array}$ \\
\hline Bebedor poco frecuente/bajo nivel & 25,5 & 66,1 & 42 \\
Bebedor moderado/bajo nivel & 8,9 & 11 & 9,7 \\
Bebedor frecuente/bajo nivel & 4,5 & 3,8 & 4,2 \\
Bebedor poco frecuente/alto nivel & 17 & 9,5 & 14 \\
Bebedor moderado/alto nivel & 23,1 & 6,2 & 16,2 \\
Bebedor frecuente/alto nivel & 5,3 & 1,9 & 3,9 \\
Bebedor consuetudinario & 15,8 & 1,4 & 10 \\
Dependencia & 13,5 & 1,9 & 8,8 \\
Problemas con la autoridad & 7,6 & 0,5 & 4,7 \\
Arresto por conducir bajo efectos del alcohol & 2,9 & 0,1 & 1,7 \\
Pérdida del trabajo & 2,7 & 0,3 & 1,8 \\
Discusión con la pareja & 14,8 & 2,1 & 9,8 \\
Reportó más de un problema & 4,5 & 0,18 & 2,8 \\
\hline
\end{tabular}

Fuente: Encuesta Nacional de Adicciones 2002. Secretaría de Salud, México.

a Las diferencias en las proporciones para todas las variables, entre hombres y mujeres, son estadísticamente significativas $(P<0.001)$.

El hecho de haber comenzado una discusión con el (la) compañero(a) como consecuencia del consumo, fue el problema asociado que se notificó con mayor frecuencia $(9,8 \%)$, seguido de problemas con la autoridad $(4,7 \%)$. En todos los casos la proporción de hombres que indicó haber tenido cualquiera de los cuatro problemas analizados fue mayor que la de mujeres (cuadro 1). Las diferencias entre géneros son notables y estadísticamente significativas $(P<0,001)$.

En lo que respecta a la demanda de servicios de atención, sólo 1,4\% de los bebedores solicitó algún tipo de ayuda por sus problemas de consumo de alcohol durante el año anterior a la entrevista: la mayoría (98\%) eran varones. Así, mientras el porcentaje de hombres que solicitaron ayuda fue de $2,1 \%$, el equivalente en las mujeres fue de sólo $0,2 \%(P<0,001)$.

Incluso cuando se analiza exclusivamente a los dependientes, el porcentaje de demanda de atención en ambos sexos sigue siendo bajo $(9,6 \%)$. Para los fines de este estudio resulta más llamativo el hecho de que las diferencias entre hombres y mujeres se intensifiquen ya que, por cada mujer dependiente que solicita ayuda, hay 49 hombres bajo la misma condición que la piden. En el cuadro 2 se especifican los porcentajes de individuos que buscaron atención de acuerdo con diferentes variables analizadas; para cada uno de los porcentajes se muestran los intervalos de confianza respectivos.

En lo que respecta a problemas asociados, las personas que perdieron un trabajo como consecuencia de la bebida son las que solicitaron ayuda en mayor proporción $(21,2 \%)$, seguidas de aquellas que tuvieron una discusión con su pareja $(8,6 \%)$.

Las variables que resultaron asociadas con la demanda de atención se muestran en el cuadro 3 , con sus respectivas razones de posibilidades (RP) e intervalos de confianza de 95\% (IC95\%). Como se evidencia, el género es una de las variables asociadas con la búsqueda de atención, y se hace evidente que los hombres tienen una probabilidad ocho veces mayor de solicitar atención que las mujeres (IC 95\%: 3,20-20,05).

En el análisis multivariado por regresión logística se reafirma la importancia de la asociación entre el género y la demanda de atención, en tanto esta variable aparece asociada significativamente, usando a las mujeres como referencia ( $\mathrm{RP}=2,93$; IC95\%: $1,12-7,66)$, aún controlando por la presencia de dependencia y por el hecho 
CUADRO 2. Porcentaje de personas que buscaron ayuda, según variables seleccionadas, México, 2002

\begin{tabular}{lcc}
\hline \multicolumn{1}{c}{ Variable } & Porcentaje de solicitantes & IC 95\% ${ }^{\mathrm{a}}$ \\
\hline Hombres & 2,1 & $1,51-2,95$ \\
Mujeres & 0,2 & $0,11-0.63$ \\
$12-17$ años & 1,3 & $0,5-3$ \\
18-34 años & 1,2 & $0,7-1,9$ \\
35-65 años & 0,9 & $0,9-2,4$ \\
Bebedores consuetudinarios & 4,4 & $2,83-6,87$ \\
Dependientes & 9,5 & $6,49-13,86$ \\
Problemas con la autoridad & 6,2 & $3,55-10,87$ \\
Arrestados por conducir bajo efectos del alcohol & 6,1 & $2,62-13,73$ \\
Perdieron el trabajo & 21,2 & $11,92-34,87$ \\
Discutieron con la pareja & 8,6 & $5,48-13,27$ \\
\hline
\end{tabular}

Fuente: Encuesta Nacional de Adicciones 2002. Secretaría de Salud, México.

a Intervalo de confianza de $95 \%$.

CUADRO 3. Variables asociadas con la demanda de atención en el análisis bivariado, México, 2002

\begin{tabular}{|c|c|c|}
\hline Variable & $\mathrm{RP}^{\mathrm{a}}$ & IC $95 \%$ b \\
\hline Género ${ }^{c}$ & 8,02 & $3,20-20,05$ \\
\hline Patrón de consumo ${ }^{d}$ & 1,57 & $1,39-1,78$ \\
\hline Dependencia & 18,38 & $10,66-31,70$ \\
\hline Problemas con la autoridad & 5,90 & $2,87-12,13$ \\
\hline Arrestado mientras consumía & 5,05 & $1,97-12,92$ \\
\hline Perdió un trabajo & 23,93 & $10,50-54,55$ \\
\hline Discusión con el/la esposo/a, compañero/a & 11,69 & $6,07-22,52$ \\
\hline
\end{tabular}

Fuente: Encuesta Nacional de Adicciones, 2002. Secretaría de Salud, México.

a Razón de posibilidades.

b Intervalo de confianza de 95\% para la RP.

${ }^{c}$ Categoría de referencia: mujeres.

${ }^{\mathrm{d}}$ Evaluada como variable ordinal.

CUADRO 4. Variables asociadas a la demanda de atención en consumidores de alcohol, según análisis por regresión logística, ${ }^{a}$ México, 2002

\begin{tabular}{lrcc}
\hline \multicolumn{1}{c}{ Variable } & $\mathrm{RP}^{\mathrm{b}}$ & Valor de $P$ & $\mathrm{IC} 95 \%{ }^{\mathrm{c}}$ \\
\hline Género $^{\mathrm{d}}$ & 2,93 & 0,028 & $1,12-7,66$ \\
Dependencia $^{\mathrm{e}}$ & 10,16 & 0,000 & $5,45-18,94$ \\
Perder el trabajo $^{\mathrm{e}}$ & 4,27 & 0,002 & $1,69-10,79$ \\
\hline
\end{tabular}

Fuente: Encuesta Nacional de Adicciones, 2002. Secretaría de Salud, México.

a Variable dependiente: demanda de atención.

b Razón de posibilidades.

c Intervalo de confianza de 95\%.

${ }^{d}$ Categoría de referencia: mujer.

e Variable dicotómica.

de haber perdido el empleo como consecuencia del consumo ( $\mathrm{RP}=4,27$; IC95\%:1, 69-10,79) (cuadro 4).

Cabe destacar que se optó por no incluir la variable "discusiones con la pareja", ya que estaba altamente correlacionada con la pérdida de trabajo ( $R P=13,4$; IC 95\%: 6,15-29,19) y con el género ( $\mathrm{RP}=7,94$; IC 95\%: 5,0-12,6), $\mathrm{y}$ éstas mostraban un mayor poder explicativo, tanto estadística como conceptualmente.

\section{DISCUSIÓN}

En consonancia con los hallazgos de estudios previos, este trabajo muestra que los varones tienen una mayor probabilidad de buscar ayuda por sus problemas de consumo de alcohol en comparación con las mujeres, incluso cuando el análisis se limita a subgrupos con alta probabilidad de requerir ayuda. Así, y a manera de ejemplo, esta relación se mantiene aún cuando se controla por otros factores como son presentar síndrome de dependencia y haber perdido el trabajo como consecuencia de la forma de beber. Lamentablemente, debido a que en este estudio el número de mujeres solicitantes de servicios es muy bajo, fue imposible realizar análisis específicos para evaluar qué variables tienen un efecto específico sobre la probabilidad de que las mujeres busquen ayuda. Sin embargo, esto puede ser objeto de estudios futuros para poner a prueba una serie de reflexiones relacionadas con los obstáculos que enfrentan las mujeres para ingresar a algún tipo de tratamiento y que estarían relacionados con barreras de tipo cultural, familiar y de los propios servicios de atención para su acceso por parte de las mujeres. Entre las dificultades de tipo cultural se encontraría la muy baja tolerancia social hacia el alcoholismo en las mujeres, con la consecuente estigmatización que ello produce. Lo anterior conlleva, por un lado y según los datos de esta encuesta y de encuestas previas (20), a que las mujeres beban con patrones de consumo de menor riesgo y presenten, en proporción, menos problemas asociados que los hombres $\mathrm{y}$, por otro lado, a que tiendan a beber en la soledad de sus hogares con menos consecuencias sociales (problemas con la policía, conducir bajo efectos del alcohol, violencia callejera) y con más dificultad para identificar y aceptar el problema y una mayor tendencia a ocultarlo y negarlo $(16,25,26)$.

Este dato se ha relacionado con las pautas socialmente establecidas, según 
roles de género para hombres y mujeres, donde el hecho de ser mujer y beber en exceso contradice el patrón cultural tradicional de una "buena madre" o una "buena esposa" (27).

Al fenómeno de la estigmatización deben sumarse las responsabilidades familiares, sobre todo con respecto al cuidado de los hijos, lo cual se ha notificado como un obstáculo muy importante para que las mujeres soliciten un servicio (18).

Asimismo, existen factores socioeconómicos que pueden actuar como barreras de acceso en las mujeres, quienes suelen tener menores niveles de ingreso que los hombres o bien son económicamente dependientes de éstos. Según la Encuesta Nacional de Ingreso y Gasto en Hogares 2004, el promedio de ingreso trimestral de los hogares cuyos jefes son mujeres es $21 \%$ menor que los hogares con jefes hombres. (28)

Finalmente, entre las barreras de acceso cabe mencionar a las vinculadas con la organización de los servicios especializados. En general, las modalidades de tratamiento no se han adecuado a las necesidades específicas de las mujeres. Entre las barreras organizativas están la composición del equipo terapéutico, la distribución geográfica y otros factores como el costo $(14,15,25)$.

La variable que se mantiene como un importante predictor después de ser controlada por el género y la dependencia, es haber perdido el empleo por la bebida, lo cual confirma el hallazgo en estudios previos en cuanto a que la presencia de problemas sociales relacionados son buenos predictores de la búsqueda de atención. En este es- tudio, el hecho de ser varón está significativamente asociado con perder un trabajo como consecuencia de la bebida. Esta asociación hace pensar que esa variable puede ser un detonante o una "señal de acción" para la búsqueda de servicios, ya que se trata de un evento muy sensible en el funcionamiento de las personas, sobre todo en los hombres, quienes en mayor proporción llevan el peso de la economía familiar, en tanto que para las mujeres los problemas de tipo afectivo o familiares son los más importantes (18). El papel del hombre como proveedor económico de la familia, aunado a una menor probabilidad de que las mujeres se expongan a la pérdida de trabajo, son variables asociadas al género que pueden estar determinando, en gran medida, tanto el patrón de consumo como la probabilidad de buscar ayuda.

De los hallazgos de este estudio se desprenden algunas implicaciones en cuanto a la organización de los servicios de salud en esta materia, al observar el fenómeno desde una perspectiva de género.

Los resultados a los que se ha arribado en este trabajo hacen evidentes los muy bajos niveles de la demanda de atención, agudizados en el caso de las mujeres. Este hallazgo podría estar relacionado, por un lado, con el ejercicio de roles de género en lo que respecta al consumo de alcohol en las mujeres y con la presión y estigmatización que surgen cuando ese consumo es excesivo, lo cual puede repercutir en la aparición de mayores obstáculos internos en las mujeres para acudir a servicios de atención. Por otro lado, no puede dejarse de lado el papel que de- sempeña la organización de los servicios de atención a personas con problemas relacionados con el consumo de alcohol ya que, si bien es cierto que a partir de la segunda mitad del siglo XX los sistemas de tratamiento han tenido importantes avances tanto en su integración como en la oferta de servicios con enfoques profesionales de respeto a los derechos humanos, mucho resta por hacer todavía para lograr un sistema de atención con suficiente cobertura y calidad en los servicios.

El conocimiento de la magnitud y de los factores asociados con la demanda de servicios de salud por consumo de alcohol y, en este caso, el énfasis en una perspectiva de género, son de utilidad para enfocar de manera más eficaz la planeación de servicios a través de programas de tratamiento diseñados para atender las necesidades específicas de las mujeres, como son la comorbilidad psiquiátrica y las alteraciones emocionales y físicas derivadas de su mayor vulnerabilidad, así como la atención a la salud reproductiva y la orientación en el cuidado de los hijos. Estas medidas deben ir acompañadas de una campaña con información clara que evite los tintes moralistas que podrían culpabilizar a las mujeres en lugar de acercarlas a los servicios especializados.

A modo de cierre, cabe considerar que si bien es bajo el porcentaje de mujeres bebedoras con patrones de consumo de alto riesgo y problemas relacionados, la generación de opciones de tratamiento diseñadas para atender las necesidades de estas mujeres es un ejercicio imprescindible e impostergable en un sistema de salud que busca mejorar la equidad en sus servicios.

\section{REFERENCIAS}

1. Smart RG, Gillies M, Brown G, Blair NL. A survey of alcohol-related problems and their treatment. Can J Psychiatry. 1980; 25(3):220-7.

2. Weisner C, Greenfield T, Room R. Trends in the treatment of alcohol problems in the US general population, 1979 through 1990. Am J Public Health. 1995;85(1):55-60.

3. Ogborne AC, DeWit DJ. Lifetime use of professional and community services for help with drinking: results from a Canadian pop- ulation survey. J Stud Alcohol. 1999;60(6): $867-72$.

4. Medina-Mora ME, Natera G, Borges G. Alcoholismo y abuso de bebidas alcohólicas. En: Observatorio Mexicano en Tabaco, Alcohol y otras Drogas. Secretaría de Salud, Consejo Nacional contra las Adicciones: México DF; 2002. Pp. 15-25.

5. Wu LT, Ringwalt CL, Williams CE. Use of substance abuse treatment services by persons with mental health and substance use problems. Psychiatr Serv. 2003;54(3): 363-9.

6. Cohen E, Feinn R, Arias A, Kranzler HR. Alcohol treatment utilization: findings from de National Epidemiologic Survey on alcohol and related conditions. Drug Alcohol Depend. 2007;12;86(2-3):214-21.

7. Hingson R, Mangione T, Meyers A, Scotch N. Seeking help for drinking problems. A study 
in the Boston Metropolitan Area. J Stud Alcohol. 1982;43(3):273-88.

8. Tucker JA. Predictors of help-seeking and the temporal relationship of help to recovery among treated and untreated recovered problem drinkers. Addiction. 1995;90(6):805-9.

9. Weisner $C$. Toward an alcohol treatment entry model: a comparison of problem drinkers in the general population and in treatment. Alcohol Clin Exp Res. 1993;17(4):746-52.

10. Finney JW, Moos RH. Entering treatment for alcohol abuse: a stress and coping model. Addiction. 1995;90(9):1223-40.

11. Hajema KJ, Knibbe RA, Drop MJ. Social resources and alcohol-related losses as predictors of help seeking among male problem drinkers. J Stud Alcohol. 1999;60(1):120-9.

12. Tucker JA, Gladsjo JA. Help-seeking and recovery by problem drinkers: characteristics of drinkers who attended Alcoholics Anonymous or formal treatment or who recovered without assistance. Addict Behav. 1993;18(5): 529-42.

13. George AA, Tucker JA. Help-seeking for alcohol-related problems: social contexts surrounding entry into alcoholism treatment or alcoholics anonymous. J Stud Alcohol. 1996; 57(4):449-57.

14. Beckman LJ, Kocel K. The treatment-delivery system and alcohol abuse in women: social policy implications. J Soc Issues. 1982;38(2): 139-51.

15. Solís LR, Medina-Mora ME. La utilización de servicios de atención para la salud mental por mujeres mexicanas. Resultados de dos encuestas nacionales. Salud Mental. 1994;(17)1: 7-10.
16. Thom B. Sex differences in help-seeking for alcohol problems. The barriers to helpseeking. Br J Addict. 1986;81(6):777-88.

17. Kaskutas LA, Weisner C, Caetano R. Predictors of health seeking among a longitudinal sample of the general population, 1984-1992. J Stud Alcohol. 1997;58(2):155-61.

18. Beckman LJ, Amaro H. Personal and social difficulties faced by women and men entering alcoholism treatment. J Stud Alcohol. 1985; 47(2):135-45.

19. Medina-Mora ME, Cravioto $\mathrm{P}$, Villatoro J, Galván F, Fleiz C, Rojas E, et al. Encuesta Nacional de Adicciones 2002. Capítulo de Alcohol. Consejo Nacional contra las Adicciones, CONADIC; Instituto Nacional de Psiquiatría Ramón de la Fuente Muñiz, INPRFM; Dirección General de Epidemiología, DGE; Instituto Nacional de Estadística, Geografía e Informática, INEGI. México. Hallado en: http://www.conadic.salud.gob.mx/pie/ena 2002.html. Acceso el 7 febrero 2008.

20. Secretaría de Salud, Dirección General de Epidemiología, Instituto Mexicano de Psiquiatría. Encuesta Nacional de Adicciones; 1990, 1994, 1998. México: Secretaría de Salud, Dirección General de Epidemiología, Instituto Mexicano de Psiquiatría; 1990, 1994, 1998.

21. Medina-Mora ME, Terroba G. Relación entre patrones de consumo de alcohol y problemas relacionados. Evaluación de su operacionalización. Reporte interno. México, D.F.: Instituto Mexicano de Psiquiatría; 1980.

22. Medina-Mora ME. Diferencias por género en las prácticas de consumo de alcohol. Resultados de un estudio llevado a cabo en la población de 18 años y más de una entidad urbana y otra rural del estado de Michoacán [tesis doctoral]. México, D.F.: Universidad Nacional Autónoma de México; 1993.

23. Medina Mora ME, Fleiz C. Alcoholismo. Abuso y dependencia de sustancias psicoactivas. En: Alarcón RD, Mazzotti G, Nicolini H, eds. Psiquiatría. 2a . ed. México, D.F.: Organización Panamericana de la Salud-El Manual Moderno; 1989. Pp. 299-365.

24. Organización Mundial de la Salud. Clasificación estadística internacional de enfermedades y problemas relacionados con la salud. 10a. revisión. V. 1. Washington, D.C.: OPS; 1995.

25. Thom B. A process approach to women's use of alcohol services. Br J Addict. 1984;79(4): 377-82

26. Pérez-López C, González L, Rosovsky H, Casanova L. La mujer en los grupos de Alcohólicos Anónimos. Anales del Instituto Mexicano de Psiquiatría. Reseña de la VII Reunión de Investigación. México: Instituto Mexicano de Psiquiatría; 1992

27. Rosovsky H, Casanova L, Pérez C. Las características de los grupos y de los miembros de Alcohólicos Anónimos. Anales del Instituto Mexicano de Psiquiatría. Reseña de la VI Reunión de Investigación. México: Instituto Mexicano de Psiquiatría; 1991.

28. Instituto Nacional de Estadística, Geografía e Informática. INEGI (2004) Encuesta Nacional de Ingresos y Gasto de los Hogares (ENIGH 2004). México: Instituto Nacional de Estadística, Geografía e Informática; 2004.

Manuscrito recibido el 2 de abril de 2007. Aceptado para publicación, tras revisión, el 18 de diciembre de 2007.

ABSTRACT Objectives. To update information available on the role that gender plays when consumers of alcohol seek help, as well as to evaluate the associations between the demand for treatment and other key variables.

The role of gender in the demand for treatment of problems associated with alcohol consumption in Mexico

Key words
Methods. Data came from 2002 National Survey on Addictions (Encuesta Nacional de Adicciones) in Mexico, which interviewed one randomly-selected individual from 12-65 years of age in each of 11252 homes. The results of this study are based on the responses of the 5406 individuals identified as consumers of alcohol. The demand for health care services was defined as seeking treatment for drinking-related problems during the last year. Alcohol consumers were defined as those people who had consumed alcohol during the last year. The national treatment-seeking prevalence rate was calculated by gender, consumption patterns, dependency syndrome, and other alcohol-related issues. In the logistical regression analyses, seeking treatment was used as the dependent variable.

Results. Of the total consumers, $1.4 \%$ sought treatment for drinking-related problems. For males, the corresponding percentage was $2.1 \%$, while for women it was $0.2 \%(\mathrm{P}<0.001)$. For each woman with an alcohol dependency who got help, there were 49 males who did so. In the multivariate analysis, gender was significantly associated, using females as the reference, even when controlling for dependency and for absenteeism at work due to excessive alcohol consumption.

Conclusions. The probability of seeking treatment is higher among males than females who are faced with cultural, family, and treatment-related barriers. Another important predictor is work absenteeism due to excess drinking. Health care planning needs to sharpen its focus through programs designed to address the specific needs of women.

Mental health services, alcohol abuse, gender, Mexico. 